\title{
Muséologies
}

Les cahiers d'études supérieures

muséologies

\section{L'exposition comme média de changement social}

\section{Mélanie Girard}

Volume 4, numéro 1, automne 2009

URI : https://id.erudit.org/iderudit/1033534ar

DOI : https://doi.org/10.7202/1033534ar

Aller au sommaire du numéro

Éditeur(s)

Association Québécoise de Promotion des Recherches Étudiantes en Muséologie (AQPREM)

ISSN

1718-5181 (imprimé)

1929-7815 (numérique)

Découvrir la revue

Citer cet article

Girard, M. (2009). L’exposition comme média de changement social.

Muséologies, 4(1), 94-107. https://doi.org/10.7202/1033534ar

\section{Résumé de l'article}

Depuis vingt ans, la communauté muséale, portée par l'évolution de la muséologie sociale durant les années 1980, s’est rapprochée de la société et s'est transformée en agent actif de changements. La conception d'expositions à caractère social est devenue pour le musée un médium de communication apte à promouvoir ses valeurs. En s'appuyant sur l'exposition Il était un toit présentée à l'Odyssée des Bâtisseurs, Mélanie Girard démontre comment les approches développées dans le domaine du marketing social peuvent enrichir le processus de conception de ce type d'exposition.
Ce document est protégé par la loi sur le droit d'auteur. L’utilisation des services d'Érudit (y compris la reproduction) est assujettie à sa politique d'utilisation que vous pouvez consulter en ligne.

https://apropos.erudit.org/fr/usagers/politique-dutilisation/ 
Article quatre

L'exposition comme média de changement social ${ }^{[1]}$

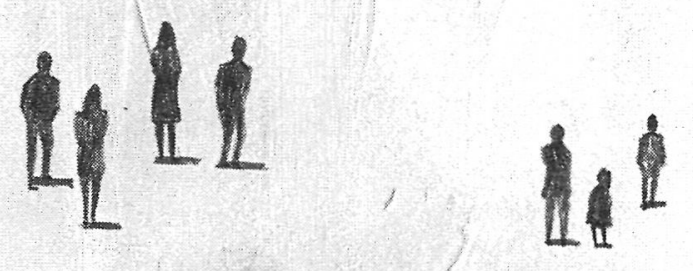


Depuis vingt ans, la communauté muséale, portée par l'évolution de la muséologie sociale durant les années 1980, s'est rapprochée de la société et s'est transformée en agent actif de changements. La conception d'expositions à caractère social est devenue pour le musée un médium de communication apte à promouvoir ses valeurs. En s'appuyant sur l'exposition Il était un toit présentée à l'Odyssée des Bâtisseurs, Mélanie Girard démontre comment les approches développées dans le domaine du marketing social peuvent enrichir le processus de conception de ce type d'exposition.

MÉLANIE GIRARD EST TITULAIRE D'UNE MAITTRISE EN HISTOIRE DE L'UNIVERSITÉ DE MONTRÉAL (2003) ET D'UN DIPLÓME D'ÉTUDES SUPÉRIEURES SPÉCIALISÉES EN MUSÉOLOGIE DE L'UNIVERSITÉ LAVAL (2007). ELLE TRAVAILLE ACTUELLEMENT COMME CHARGÉE DE PROJET AU RENOUVELLEMENT DE L'EXPOSITION PERMANENTE DU MUSÉE DES URSULINES DE QUÉBEC. DEPUIS L'AUTOMNE 2007, ELLE POURSUIT DES ÉTUDES DOCTORALES EN COMMUNICATION PUBLIQUE À L'UNIVERSITÉ LAVAL ET SES RECHERCHES PORTENT SUR LES EXPOSITIONS MISSIONNAIRES. [melanie.girard.3@ulaval.ca] 
Cet article est tiré de GIRARD, Mélanie. L'exposition comme média de changement social. Québec: Université Laval, essai dirigé pour le diplôme d'études supérieures spécialisées en muséologie (DESS), 2007.

[2]

FLEMING, David. "The Museum as Social Enterprise”. INTERCOM Annual Meeting 2006 Taipei, 2 November 2006. The First Stephen E Weil Memorial Lecture. <http://www.intercom. museum/Taiwan2006a.html> (consulté en avril 2009).

International Workshop on New Museology, 1987. Cité dans MERLEAU-PONTY, Claire et Jean-Jacques EZRATI. L'exposition, théorie et pratique. Paris: L'Harmattan, 2005, p. 18.
I have no doubt that museums are powerful agents of social change, and the more we look at the ways in which they are capable of operating, the more convincing a case we can make.

David Fleming ${ }^{[2]}$.

"The ways in which [museums] are capable of operating", voilà ce qui nous intéresse dans cet article. Plus précisément, nous nous pencherons sur l'exposition. Celle-ci sera ici perçue dans une perspective médiatique et communicationnelle et ce, non seulement comme véhicule d'information, mais comme participant au changement social. Ce qui nous préoccupe, en fait, est la façon dont le musée peut s'y prendre pour sensibiliser ses publics à un enjeu social et encourager un changement d'attitude ou de comportement de la part du visiteur. Dans cet article, nous tenterons de mettre de l'avant que les approches développées (et éprouvées) dans le domaine du marketing social peuvent enrichir le processus de conception d'une exposition à caractère social.

\section{Le rôle social du musée}

De plus en plus, la communauté muséale est appelée à prendre part aux enjeux de société, à jouer un rôle actif et à participer au changement social. Changement social réfere ici à tout changement de valeur, de comportement, observable dans un groupe défini et à caractère durable.

Le discours de la nouvelle muséologie, développé dans les années 1980, s'inscrit dans cette tendance à privilégier la dimension sociale et politique, entendue au sens de civique, public: « La muséologie doit avoir pour but, la libération, les progrès et les transformations de la société par la prise de conscience et la participation de la population ${ }^{[3]}$.»

Cette nouvelle muséologie qui privilégie le rôle social du musée, sans s'être répandue à l'ensemble du champ muséal, se traduit par une préoccupation grandissante pour les visiteurs perçus comme des citoyens. À ce 
titre, les musées inscrivent à leur programmation des thématiques en lien avec les enjeux de société.

[U]ne des retombées [du mouvement de la nouvelle muséologie] est le développement d'une muséologie expressive qui assume résolument sa dimension communicationnelle et donne lieu à un style d'exposition thématique où sont proposés des points de vue revendiquant leur part de subjectivité sur les rapports à la réalité sociale et naturelle, et appelant de la part du visiteur un positionnement impliqué ou critique ${ }^{[4]}$.

Cette mouvance vers une muséologie sociale se perçoit aujourd'hui au Canada de même qu'aux États-Unis, alors qu'on associe le concept de développement durable (sustainability) à la culture et au milieu muséal. En 2000, le Working Group on Museums and Sustainable Communities est mis sur pied:

It is focusing its efforts on engaging the museum community in Canada in a process of awareness, reflection, learning, sharing of knowledge and experience, capacity-building and action related to their role in creating a culture of sustainability ${ }^{[5]}$.

\section{Le marketing social}

Défini comme une forme de communication visant à susciter le changement dans les perceptions, les attitudes ou les comportements du public, le marketing social s'apparente au type d'exposition qui nous intéresse ici. À cet égard, nous jugeons pertinent d'établir des liens entre la démarche entourant l'élaboration d'un programme de marketing social et la mise en œuvre d'un projet d'exposition à des fins de sensibilisation et de changement social. Avant toute chose, il convient d'expliquer en quoi consiste le marketing social. En fait, il s'agit d'une application des techniques et des outils du marketing commercial à des problématiques sociétales, culturelles ou environnementales.

L'idée d'appliquer les techniques du marketing commercial à des causes sociales remonte aux années 1950 et 1960. L'expression " marketing social» (social marketing) est utilisée pour la première fois en 1971 par Philip Kotler et Gerald Zaltman ${ }^{[6]}$. Ils le définissent alors comme: « the design, implementation and control of programs calculated to influence the acceptabilities of social ideas and involving considerations of product planning, pricing, communication, distribution and marketing research ${ }^{[7]}$.

Le marketing social se distingue cependant du marketing commercial par son objet : attitudes et comportements remplacent ici produits et
[4]

LE MAREC, Joëlle. «Des étagères enfantines aux musées virtuels...". MédiaMorphose, $n^{\circ} 9,2003$, p. 23.

[5]

THE WORKING

GROUP ON MUSEUMS

AND SUSTAINABLE

COMMUNITIES. Museums

and Sustainable Communities.

Resource Document <http://

www.cultural-connections.org/

programs/meeting_notes/

feb2004_suggested_reading.

pdf $>$ (consulté en avril 2009).

[6]

Dans un article intitulé « Social

Marketing: an Approach to

Planned Social Change», publié dans le Journal of Marketing, vol. 35, juillet 1971, p. 3-12.

[7]

Cité dans: MACFADYEN, Lynn, Martine STEAD et Gerard HASTING. A Synopsis of Social Marketing. <http://www. ism.stir.ac.uk/pdf_docs/social_ marketing.pdf $>$ (consulté en avril 2009). 
KOTLER, Philip et Nancy R. LEE. Social Marketing. Influencing Behaviors for Good. Los Angeles: Sage Publications, 2008, p. 13.

[9]

LE MAREC, Joëlle. Publics et musées. La confiance éprouvée. Paris: L'Harmattan, 2007, p. 135.

[10]

L'exposition /I était un toit. Petite histoire de l'architecture domestique dans l'est du Canada a été inaugurée en juillet 2006 à l'Odyssée des Bâtisseurs (Alma). Nous avons agi, pour la réalisation de cette exposition, à titre de chargée

de projet à l'occasion d'un stage au DESS en muséologie (Université Laval). Ce projet a été réalisé avec Patrimoine Canada et le Musée virtuel du Canada (MVC), sous la direction de la Société d'histoire du Lac-Saint-Jean, à l'aide de l'expertise de son service d'aide-conseil en rénovation patrimoniale (SARP) et en collaboration avec le laboratoire de muséologie et d'ingénierie de la culture (LAMIC) de l'Université Laval. services. En outre, dans le cas du marketing commercial, il s'agit de faire du profit; dans celui du marketing social, il s'agit plutôt de "faire profiter " l'individu et la société en général puisque ce n'est pas là une activité mercantile. Bref, il n'est pas ici question de vendre des produits ou des services, mais plutôt de promouvoir des attitudes ou des comportements pour le bénéfice de l'individu et de la société ${ }^{[8]}$.

Au Québec, c'est le publicitaire Jacques Bouchard, président-fondateur du Publicité Club de Montréal, qui a été le premier à faire la promotion du marketing social dans son ouvrage intitulé L'autre publicité: la publicité sociétale (Héritage, Saint-Lambert, 1981). La publicité sociétale est souvent utilisée par les instances gouvernementales pour corriger un comportement jugé déficient, par exemple pour lutter contre le tabagisme, contre la vitesse ou l'alcool au volant, ou encore pour promouvoir l'activité physique.

\section{Les stratégies du marketing social appliquées à l'élaboration}

\section{d'une exposition}

D'aucune façon, il n'est question ici d'appliquer une quelconque logique marchande au musée ou à l'exposition. D'ailleurs, comme le fait remarquer Joëlle Le Marec, «les publics des musées ne se sentent pas consommateurs des biens et services proposés par un offreur. Tout au contraire, la construction du statut de visiteur et de la pratique de visite [...] s'effectue contre le modèle de l'échange marchand ${ }^{[9]}$."

Nous verrons plutôt dans quelle mesure les stratégies du marketing social peuvent enrichir la conception d'expositions visant à encourager un changement de comportement ou d'attitude chez les visiteurs. Pour ce faire, nous aurons recours à un exemple concret, à savoir la réalisation de l'exposition itinérante intitulée Il était un toit, à laquelle nous avons participé, qui visait à sensibiliser les visiteurs au patrimoine bâti résidentiel et à favoriser la pratique d'une rénovation à caractère patrimonial. Cette exposition a été présentée au parc thématique l'Odyssée des Bâtisseurs à Alma (Québec, Canada) ${ }^{[10]}$.

Précisons que c'est une réflexion a posteriori qui nous amène à soutenir qu'une connaissance des approches du marketing social aurait pu rendre encore plus fort le message que nous voulions alors transmettre. Nous espérons ainsi que notre réflexion pourra profiter à quiquonque entreprendra une démarche similaire. 
Dans l'optique d'appliquer les outils du marketing commercial à des fins de sensibilisation et de changement social, il conviendra d'abord de cerner les quatre éléments qui sont à la base du marketing, communément appellés le «marketing-mix» : le produit, le prix, le positionnement et la promotion.

\section{Le produit}

Il s'agit du comportement ou de l'attitude que l'on cherche à promouvoir. C'est aussi le bénéfice, les avantages que les visiteurs peuvent retirer de ce nouveau comportement (mieux-vivre, mieux-être en société, environnement plus sain, etc.).

Dans le cas de l'exposition Il était un toit, le produit était en fait la démarche de rénovation patrimoniale. Cette exposition avait comme objectifs de sensibiliser les publics au patrimoine bâti résidentiel et de promouvoir un souci de rénovation qui tienne compte des aspects patrimoniaux. Lorsque vient le temps d'adapter leur maison à leurs besoins, les occupants ne se préoccupent pas nécessairement de conserver son aspect patrimonial architectural. Il s'agissait donc de mettre en valeur le patrimoine résidentiel canadien, d'offrir une réflexion sur ce qui advient de cet héritage, puis de présenter et d'expliquer aux visiteurs la démarche de rénovation patrimoniale. En outre, il s'agissait de faire ressortir que la préservation de l'aspect architectural des résidences bénéficie non seulement aux propriétaires, mais aussi à l'image et à l'environnement des municipalités.

\section{Le prix}

Le prix constitue le coût total que représente l'adoption de la nouvelle attitude ou du nouveau comportement. Outre ce qu'il en coûte au public pour visiter l'exposition (il doit se déplacer, payer le prix d'entrée, etc.), le prix est constitué de l'effort, de ce que le visiteur doit laisser tomber pour adopter le comportement, pour changer d'attitude. Pour déterminer ce prix le plus justement possible il s'avère essentiel de bien connaître ses publics cibles, leurs valeurs, leurs attitudes, etc.

Dans l'élaboration d'un programme de marketing social, l'analyse du ou des publics cibles est déterminante et s'effectue dès que les objectifs ont été définis. Le programme sera d'autant plus efficace s'il prend appui sur une solide connaissance du ou des publics cibles ${ }^{[11]}$. Il s'agit d'effectuer des recherches afin de recueillir des données d'ordre démographique telles que l'âge ou le niveau d'instruction et des données psychographiques comme les
[11]

SOCIAL MARKETING INSTITUTE. <http://www. social-marketing.org/sm.htm $\triangleright$ (consulté en avril 2009). 
[12]

SANTÉ CANADA. Marketing social. <http://www.hc-sc. gc.ca/ahc-asc/activit/marketsoc/index_f.htm $>$ (consulté en avril 2009).

[13]

André GIORDAN, cité dans DAIGNAULT, Lucie. "La démocratisation des musées quand les visiteurs deviennent aussi des acteurs". Colloque "Musée, acteur politique", 2 octobre 1997 (non publié), p. 11.

[14]

CHAUMIER, Serge. "Les méthodes de l'évaluation muséale. Quelques repères au sujet des formes et des techniques". La Lettre de I'OCIM, $n^{\circ} 65,1999$, p. 16.

[15]

DAIGNAULT, op. cit., p. 5. croyances, les valeurs, les opinions ${ }^{\left[{ }^{[2]}\right]}$. Pour que le message soit compris des visiteurs, il est essentiel de chercher à comprendre ce qui motive les publics cibles dans leurs choix.

[L]es apprenants possèdent, avant d'aborder toute action d'enseignement ou de médiation, des idées ou des façons de raisonner spécifiques sur les thèmes abordés. Ces conceptions comme on les appelle en didactique ont une certaine stabilité, et la sensibilisation, l'acquisition d'une démarche de pensée ou l'apprentissage d'une connaissance en dépendent complètement. Si l'on en tient pas compte, ces conceptions se maintiennent et le savoir proposé glisse généralement à la surface sans même imprégner les apprenants ${ }^{[13]}$.

Ce type de recherche sur les publics cibles existe dans le milieu muséal, quoique ce ne soit pas nécessairement une pratique répandue. Cette évaluation préalable « consiste à mieux connaître le niveau de connaissance d'un public potentiel, les imaginaires sociaux, les représentations sociales, liées à un domaine donné, afin de partir de ces données pour conduire le public là où on entend le mener " "[4].

Au Musée de la civilisation de Québec, de telles recherches ont été menées lors de la mise en œuvre de certains projets d'expositions à caractère social, sur des sujets comme la mort, la drogue et l'environnement. Ces recherches préalables servent entre autres à évaluer l'intérêt du public pour un thème, ses idées préconçues, les attentes des visiteurs quant au traitement du sujet (thèmes qui devraient selon eux être abordés, ton qu'il conviendrait d'employer, etc.). Par ailleurs, Lucie Daignault du Musée de la civilisation souligne qu' « un autre objectif des études préalables, bien entendu lorsqu'il s'agit d'exposition sociale, est de voir s'ils sont réceptifs aux messages à caractères sociaux qui visent la modification des attitudes et des comportements des visiteurs " ${ }^{[151}$. Il ne s'agit donc pas seulement de cerner les publics cibles, mais d'apprendre à mieux les connaître pour optimiser la démarche de communication. Tout l'art du marketing (et une bonne partie du discours de l'exposition) consiste à faire valoir le bénéfice que les visiteurs sont susceptibles de retirer, bref de faire en sorte que le coût soit inférieur au bénéfice. C'est ce qui sera au cœur de l'argumentation, le message principal de l'exposition.

Dans le cas de l'exposition Il était un toit, nous n'avons pu réaliser d'évaluation préalable, mais avons bénéficé de l'expertise et de l'expérience du Service d'aide à la rénovation patrimoniale (SARP). Au cours des dernières années, l'équipe du SARP a effectué de nombreuses consultations et rencontré beaucoup de gens; elle connaît donc bien la clientèle ciblée, ses questionnements, ses priorités, son sens pratique. L'équipe du SARP a en conséquence acquis une expérience solide pour expliquer la rénovation 
patrimoniale afin que le concept soit bien compris et pour devancer les objections et les interrogations. Par exemple:

- Beaucoup de gens ne croient pas que leur maison appartienne à un style architectural en particulier. Il convenait donc de leur donner des clés pour qu'ils puissent reconnaître certains styles architecturaux.

- Le mot " patrimonial " pouvant laisser croire qu'il s'agit de restauration, certains estimaient que l'entreprise pourrait s'avérer coûteuse. Il s'agissait alors d'expliquer clairement ce en quoi consistait la rénovation patrimoniale et d'en montrer des exemples.

\section{Le positionnement}

Le positionnement réferre $\mathrm{au}(\mathrm{x}) \operatorname{lieu}(\mathrm{x})$ et à la façon de rejoindre le ou les publics. François Lagarde, expert-conseil en marketing social, souligne qu'un des critères d'évaluation d'une campagne sociétale est que «le messager [soit] perçu [par le public] comme une source crédible d'information " ${ }^{[16]}$.

Le musée est une institution qui a la confiance du public. Lorsqu'il s'agit de promouvoir une attitude ou un comportement, l'institution muséale doit se poser certaines questions d'ordre éthique afin de conserver cette crédibilité qu'on lui accorde. D’emblée, il est important de se demander si l'exposition est le bon média pour aborder ce thème (pour promouvoir ce "produit"). Par ailleurs, il faut aussi se demander si le thème de l'exposition s'intègre dans la mission et le mandat de l'institution où celle-ci est présentée.

La thématique de l'exposition Il était un toit s'intégrait tout à fait à la mission et au mandat de l'Odyssée des Bâtisseurs qui a entre autres pour buts de préserver et de mettre en valeur le patrimoine résidentiel canadien et de sensibiliser les gens à l'importance de conserver et de protéger le patrimoine bâti. En cela, l'exposition est en quelque sorte le prolongement du travail effectué par le SARP, la mise en exposition du service de consultation.

La cohérence entre la mission de l'institution et la thématique de l'exposition est d'ailleurs indispensable à la bonne réception par les publics cibles du message de l'exposition. De surcroît, par souci éthique, l'institution doit aussi s'interroger sur les raisons qui motivent ce choix de thème d'exposition.

Si jamais des institutions muséales en venaient à choisir des thèmes tel [sic] qu' " environnement " en fonction de leur " succès " médiatique, avec l'objectif principal de faire grimper les chiffres de fréquentation et
[16]

LAGARDE, François. «Une campagne sociétale, ça change pas le monde, sauf que... . Présentation PowerPoint à l'occasion de la journée des communications gouvernementales, $1^{\text {er }}$ novembre 2005. $<$ http://forumdc.gouv.qc.ca/ journee05/Publicite_societale. pd> (consulté en avril 2009). 
de multiplier les retombées médiatiques, une erreur d'appréciation serait commise, car non seulement l'institution ne saurait répondre aux aspirations suscitées par un tel thème, mais bien plus, l'institution muséale se trahirait comme ayant, elle aussi, perdu sa spécificité sociale et culturelle, pour être à son tour absorbée dans le marché ${ }^{[17]}$.

\section{La promotion}

Cet élément du « marketing-mix» consiste à assurer le rayonnement du produit (du comportement). Le marketing social utilise souvent les médias de masse comme la télévision et les journaux pour véhiculer son message. La particularité du média exposition est que les gens doivent se déplacer pour entendre le message. Le défi consiste alors, d'une part, à attirer les gens - à

LE MAREC, Joëlle. Le Visiteur en représentations. L'enjeu des évaluations préalables en muséologie. Thèse de doctorat en sciences de l'information et de la communication. Saint-Étienne: Université Jean Monnet, 1996.

[18]

Cette borne interactive a été réalisée par la firme Idéeclic qui a également produit une version virtuelle de l'exposition accessible sur le site du Musée virtuel du Canada.

[19]

SANTÉ CANADA, op. cit les mobiliser - pour qu'ils viennent voir cette exposition et, d'autre part, à faire en sorte qu'ils y restent le plus longtemps possible. C'est là que l'exposition acquiert une certaine originalité par rapport au marketing social diffusé dans les médias de masse et que l'on doit tabler sur le caractère particulier de l'exposition pour offrir aux visiteurs une expérience. Plusieurs éléments contribuent à la spécificité de l'exposition: sa réalité tridimensionnelle, le fait que le visiteur y déambule à son rythme, qu'il crée son propre parcours, qu'il la visite parfois en compagnie d'autres individus avec qui il peut échanger, interagir. Cette question de la promotion concerne en fait la façon de mettre en valeur l'attitude/le comportement, les stratégies de communication, le choix des objets, les moyens muséographiques, de même que la mise en espace de l'exposition.

L'exposition Il était un toit mise sur un parcours à saveur historique, alliant cognition, réflexion et action, et amenant le visiteur du début de la colonie jusqu'à aujourd'hui, où celui-ci se voit devant le défi que représente un parc industriel canadien vieillissant. Une solution est possible : la rénovation patrimoniale. Le côté plus personnel de la maison, le lien qui unit un individu, une famille, à sa maison, fait office de porte d'entrée et de sortie à l'exposition. Celle-ci offre également un exercice de simulation de rénovation patrimoniale ${ }^{\text {[181 }}$. C'était là l'occasion, pour le visiteur, de " passer à l'action ". Après avoir pris connaissance de la problématique, il est invité à faire des choix, selon le processus du SARP. Il était important pour nous qu'en plus de voir des exemples de rénovation patrimoniale, le visiteur en fasse lui-même l'essai, par simulation.

En bref, un bon programme de marketing social c'est: «le BON produit, au BON endroit, au BON moment avec la BONNE promotion " ${ }^{[19]}$. Le tableau 1 résume le tout en termes muséologiques. 
TABLEAU 1 :

Questions à se poser lors de la mise en œuvre d'une exposition visant

à contribuer au changement d'attitudes ou de comportement

LE BON PRODUIT AU BON ENDROIT

- Quelles sont les raisons qui poussent l'institution à traiter cette thématique?

- Est-ce que l'exposition est le bon média pour promouvoir cette attitude/ ce comportement?

- Est-ce que la thématique de l'exposition s'inscrit dans la mission et le mandat de l'institution qui la présente?

AU BON MOMENT

- Est-ce que le public est intéressé par cette thématique?

- En quoi la thématique est-elle un sujet d'actualité?

AVEC LA BONNE PROMOTION

- Quelle expérience compte-t-on offrir aux visiteurs?

- Comment mettre en valeur l'attitude/le comportement?

\section{Un objectif : agir sur le comportement}

On aurait tort d'affirmer que l'objectif de ce type d'exposition consiste à modifier les attitudes ou les comportements. Le changement social est un processus qui s'effectue dans le long terme et qui, de surcroît, n'est tout simplement pas mesurable. Par ailleurs, le musée est un acteur social parmi d'autres et c'est en fait l'action combinée de ces différents acteurs qui provoque le changement social. Tout au plus le musée peut-il contribuer au changement d'attitude et/ou de comportement et participer au changement social. Comme objectifs corrolaires, nous retrouvons:

- favoriser une prise de conscience par rapport à un enjeu X;

- donner aux visiteurs les moyens, les outils leur permettant de modifier leurs attitudes et/ou leurs comportements vis-à-vis cet enjeu;

- susciter une réflexion sur les conséquences de nos actions individuelles et collectives;

- à ces objectifs de base s'ajouteront, selon le cas, des objectifs plus spécifiques en lien avec la thématique, la collection à mettre en valeur et l'expérience à faire vivre au public.

Le but premier n'est pas ici d'éduquer, mais bel et bien de contribuer au changement d'attitude et/ou de comportement. L'éducation est plus un moyen, parmi d'autres, qu'une fin en soi. Car «l'information ne peut à 
elle seule provoquer le changement " ${ }^{[20]}$. Parfois, le manque d'information n'est pas ce qui fait obstacle au changement de comportement ou d'attitude. Lucie Daignault, en discutant de la mission sociale du Musée de la civilisation de Québec, parle d'une «pédagogie de la sensibilisation, alliant à la fois connaissance, conscience, réflexion et action " ${ }^{[21]}$.

Nous avons schématisé les différentes composantes du changement social que nous résumons en trois mots : conscientiser, responsabiliser et sensibiliser (ill. 1).

Conscientiser: « Faire que quelqu'un, un groupe, prenne conscience des problèmes politiques, sociaux, culturels qui se posent à lui " ; "être pleinement conscient d'une condition, d'une situation ou d'un phénomène social " "22]. Il s'agit ici d'attirer l'attention sur une situation, d'informer les visiteurs des différents tenants et aboutissants. La conscientisation agira sur les connaissances du visiteur et sur sa volonté d'agir. grand dictionnaire terminologique : <www.granddictionnaire.

SOCIAL CHANGE MEDIA.

The Seven Doors Social Marketing Approach. <http:// media.socialchange.net.au/ strategy $>$ (consulté en avril

[24]

Tiré du Petit Robert.

Responsabiliser: Nous avons traduit le mot anglais empowerment (feeling of confidence that you can be a cause of genuine change) ${ }^{[231}$ par " responsabiliser ". Il s'agit ici d'abord de faire en sorte que les visiteurs sentent qu'ils ont le pouvoir de changer quelque chose, que leur action individuelle a des conséquences. Ensuite, il faut les convaincre du bénéfice relié à l'adoption d'une nouvelle attitude, d'un nouveau comportement, que leur action individuelle va faire une différence. C'est pourquoi le ton utilisé ne doit pas être alarmiste. Il convient de lier le " problème" aux solutions potentielles.

Sensibiliser : «Rendre concret "; rendre sensible, pénétrer la sensibilité ${ }^{(24)}$. On ajouterait: faire appel aux sens et au sensible. Il est ici question de passer de l'abstrait au concret, de la théorie à la pratique. Il s'agit de donner les outils aux visiteurs pour qu'ils puissent modifier leur attitude, leur comportement au quotidien. Il convient de rendre les solutions applicables au quotidien. Idéalement, cela passe par la simulation, par l'agir.

Par ailleurs, lorsqu'on parle de faire appel aux sens, on réfere non seulement aux cinq sens, mais aussi à l'aspect plus émotif de l'être humain. Parce que l'émotion favorise un certain transfert de connaissance.

Comme nous avons pu le constater, l'optimisation de la démarche de communication d'une exposition à caractère social passe nécessairement par une solide connaissance des publics cibles. La prise en compte du public est d'autant plus importante étant donné l'objectif de ce type d'exposition qui n'est pas strictement de véhiculer, de transmettre de l'information, mais d'agir comme média de changement social, d'agir sur le public, sur ses connaissances, sur sa volonté et sa capacité d'agir. Il s'agit de faire réagir et agir les visiteurs: les conscientiser, les responsabiliser et les sensibiliser en vue de favoriser l'adoption d'une nouvelle attitude ou d'un nouveau comportement. 
ILL. 1:

Composantes du changement social.

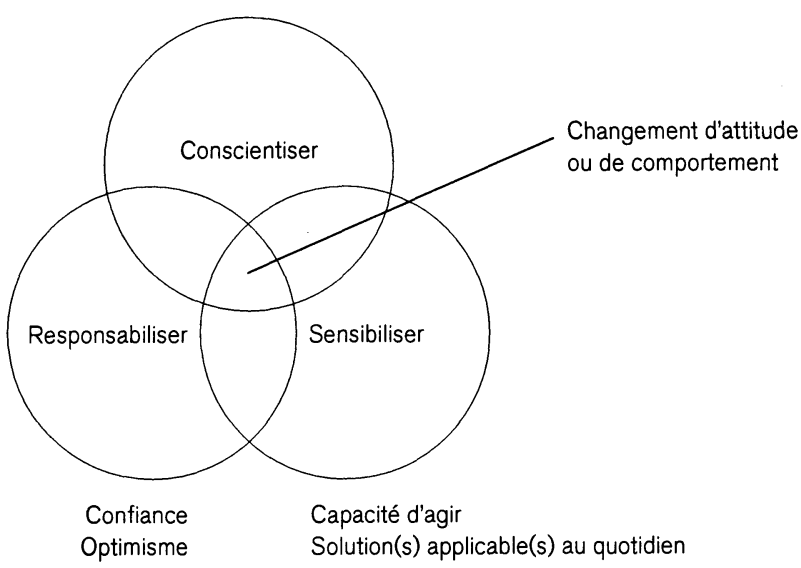

\section{Conclusion}

En comparaison avec les médias de masse, où sont diffusés des messages issus du marketing social, l'exposition se distingue, notamment, par l'implication du visiteur. Cette implication est d'abord une immersion au sens où le visiteur est - physiquement - au cœur du dispositif communicationnel, en présence d'artefacts, d'œuvres d'art, de photos, de vidéos, etc. Puis, en déambulant dans l'exposition à son rythme, en créant son propre parcours, le visiteur a aussi une part importante dans la construction de sens. Espace d'ouverture, de réflexion et non de contraintes, l'exposition, comme média de changement social, n'impose pas une nouvelle attitude ou un nouveau comportement à ses publics, elle propose. Parce qu'elle offre une véritable expérience aux visiteurs, l'exposition va au-delà d'une campagne de publicité sociétale.

Par le biais du marketing social, une expertise est développée en matière de changement d'attitude ou de comportement dès les années 1950. C'est pourquoi nous estimons que les stratégies de communication de l'exposition à caractère social gagneraient en force à s'inspirer des outils - éprouvés - du marketing social. Commele fait remarquer Roland Arpin, "On ne traite pas de thématiques sociales comme on traite de la culture matérielle. En considérant les thématiques sociales, le Musée s'éloigne des institutions de mémoire [...] et se rapproche des milieux de la communication " ${ }^{2251}$. Dans le but d'agir comme média de changement social, les muséologues ont donc tout intérêt à conjuguer la mise en œuvre de moyens muséographiques diversifiés et les approches du marketing social. Le recours à ces approches vient renforcer les stratégies de communication de l'exposition qui, à sa manière, participe au changement social. 


\section{Summary}

\section{The Exhibition as a Medium of Social Change}

[Translated by Sofia Arenzon]

[26]

This enternship took place from January to May 2006.

The exhibition /l était un toit. Petite histoire de l'architecture domestique dans l'est du Canada (Once upon a roof. $A$ short history of house architecture in Eastern Canada), inaugurated in July 2006, was presented at l'Odyssée des Bâtisseurs (Alma, Québec) through December 2007, when it left on a tour.

[27]

"Social marketing seeks to influence social behaviors not to benefit the marketer, but to benefit the target audience and the general society." Source: Website of Weinreich Communications, a social marketing consulting firm. <http:// www.social-marketing.com/ Whatis.htmD.

This article examines the exhibition as a means to communicate and to raise awareness about a social issue, with the goal of encouraging a change in the visitor's attitude or behaviour. This analysis is preceded by a case study, a handson experience during a museology internship ${ }^{[26]}$ (Laval University). It describes the creation of a travelling exhibition, intended to raise awareness about built residential heritage and to promote the integration of concepts pertaining to heritage restoration.

Our goal is to identify and to define this particular type of exhibition: firstly, in order to make it recognizable; secondly, in order to provide a few thoughts on designing such an exhibition.

That type of exhibition partly falls within the scope of a socially-oriented museology. The museological community is increasingly challenged to take part in social issues. While it has not yet spread globally, the trend to favour the social role of the Museum is reflected in a growing concern with the visitors who are perceived as citizens. Hence museums are adding socially relevant subjects to their programming. The social role of the museum is partly based on its potential to communicate and to educate the public, notably through exhibitions.

Besides bringing attention to a social issue, the type of exhibition analyzed here strives to provide visitors with tools and means that encourage them to change their attitude or behaviour. It thus links a "problem" with potential solutions: it links thought with action.

In this article our objective is to see how social marketing, which consists in applying commercial marketing techniques and tools to social, cultural, or environmental issues, so as to push for change in perceptions, attitudes, and behaviours ${ }^{[27]}$, can be applied to the medium of the exhibition. We will therefore linger on the link between designing an exhibition that strives to promote certain behaviours in the visitor and elaborating a social marketing program. Social marketing strategies cannot be strictly applied to the exhibition. Rather, we will look for information relevant to the field of museology, particularly to the art of the exhibition. 
Through the marketing mix, a series of questions will help us define the subject, the nature, and the formulation of the intended message, as well as a few ethical issues that analyze the very choice of the subject and how it relates to the mission and mandate of the institution.

Therefore, this "social marketing strategy" should not be limited to conveying information. It should also raise consciousness, awareness, and a sense of responsibility. In other words, it needs to bring attention to an "issue" and explain its various aspects; it needs to provide food for thought about the consequences of individual action, offer concrete "solutions," and demonstrate how they can be applied on a daily basis. A deep knowledge of the target audience is necessary to optimize this communication task.

The use of social marketing tools and techniques sheds a new light on the exhibition's communication strategies, which can participate in social change in a creative and original way. 